\title{
ECONOMÍA SOLIDARIA EN LA FRONTERA NORTE DE MÉXICO: LA EMERGENCIA DE ALTERNATIVAS DE DESARROLLO LOCAL Y REGIONAL
}

SOLIDARITY ECONOMY IN THE NORTHERN BORDER OF MEXICO: THE EMERGENCY OF ALTERNATIVES OF LOCAL AND REGIONAL DEVELOPMENT

CAROLINA ROSAS HEIMPEL ${ }^{1}$

\section{RESUMEN}

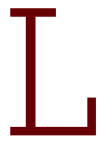
a exploración de la economía solidaria cobra cada día más relevancia cuando hablamos de aspirar a una sociedad justa, equitativa y solidaria. Frente al desalentador panorama que se vive en la frontera norte de México surge la necesidad de reflexionar sobre prácticas alternativas de desarrollo local y regional que busquen el bien común. La necesidad principal de los habitantes de Ciudad Juárez, además de la seguridad, es de índole económica: la necesidad de empleo. Mas no solo una fuente de trabajo, sino mejores condiciones laborales, menos incertidumbre y mejor salario (Padilla, Olivas, \& Alvarado, 2014), ya que las actuales condiciones de trabajo son sumamente precarias, debido

1 Universidad Autónoma de Ciudad Juárez, México. Correo electrónico: rosasheimpel. caro@gmail.com 
a que explotan, agotan y desechan a los seres humanos a favor de la productividad y los bajos costos (Jusidman y Almada, 2008). Actualmente en Ciudad Juárez $75 \%$ de la población vive en pobreza; la mayoría de los ciudadanos viven con un ingreso que no permite cubrir sus necesidades básicas. En este contexto están surgiendo saberes, prácticas y actores de transformación social que proponen otras formas de hacer economía para satisfacer sus necesidades, así como para el desarrollo de las comunidades fronterizas. Ejemplo de ello son la cooperativa Mujeres de Esperanza y Fe, cooperativa Servicio Verde, colectivo Chopeke y el banco de tiempo Doña Ana Communities United y el de Ciudad Juárez. Un acercamiento a estas experiencias muestra los retos y dificultades que las iniciativas presentan y nos permite documentar y comprender las posibilidades para definir los principios de acción en la búsqueda del bien común y el buen vivir en la frontera entre México y Estados Unidos.

Palabras clave: economía solidaria, economía social, cooperativas, frontera norte.

\section{ABSTRACT}

The exploration of the solidarity economy is more important each day when we talk about an aspiration to have a society with justice, equality, and solidarity. In front of a discouraging scenario in the northern of Mexico arise the need to reflection about how to practice the alternatives of local and regional development looking for a common good. The principal need for the citizens of Ciudad Juárez, more than a question of security, is a matter of economics questions. Not only the source of work is necessary. It's important to have a better labor conditions and the certain to retain the employment and better wages (Padilla, Olivas, \& Alvarado, 2014) as the actual conditions of a border employees is so much precarious and the humans beings are exploited looking for increase productivity and low cost for the products. And then, exhausted, they are thrown away as waste (Jusidman, \& Almada, 2008). In Ciudad Juárez $75 \%$ of the population live in poverty and most citi- 
zens obtain salaries that are not enough for the food basket and others familiars demands. In this context, are emerging practices, knowledge and actors like cooperativa Mujeres de Esperanza y Fe, cooperativa Servicio Verde, colectivo Chopeke, and banco de tiempo Doña Ana Communities United and that of Ciudad Juárez. An approach to these experiences shows the challenges and difficulties that arise and allows us to document and understand the possibilities to define the principles of action in the search of the common good and better good living in the border between Mexico and the United States.

Keywords: solidarity economy, social economy, cooperatives, Northern border.

\section{INTRODUCCIÓN}

Ante el contexto de violencia extrema y pobreza que se vive en la frontera entre México y Estados Unidos surgen saberes, prácticas y actores de transformación social que proponen una economía alternativa para satisfacer sus necesidades, así como para el desarrollo de las comunidades fronterizas. Si bien las condiciones sociales, políticas y económicas de la frontera norte entre México y Estados Unidos han creado un ambiente hostil que se caracteriza por reproducir prácticas basadas en valores como el egoísmo, la competencia, el individualismo y el afán de lucro, ello mismo genera la urgencia de crear alternativas de desarrollo local y regional.

Ejemplo de ello son iniciativas como la cooperativa Mujeres de Esperanza y Fe, cooperativa Servicio Verde, colectivo Chopeke y el banco de tiempo Doña Ana Communities United (DACU) y el de Ciudad Juárez. Para su análisis nos valimos de la sociología de las emergencias, propuesta que plantea la necesidad de una ampliación simbólica de los saberes, prácticas y agentes con el fin de identificar en ellos las tendencias del futuro (lo todavía no). Ello con un doble objetivo: "por un lado, conocer mejor las condiciones de posibilidad de la esperanza; y por otro, definir principios de acción que promuevan las realización de esas condiciones" (Santos, 2010, p. 47). En este sentido, entendemos que las prácticas que 
aquí presentamos todavía no tienen la magnitud ni el impacto político y económico que transformen nuestras comunidades o que derriben los muros que las separan. Sin embargo, analizadas desde el enfoque de la sociología de las emergencias podemos identificar en ellas las tendencias del futuro. Es por ello que aquí presentamos prácticas de economía social y solidaria que, si bien muchas de las veces son acciones pequeñas que todavía no logran cubrir las necesidades básicas de la población, sí están en la búsqueda colectiva del camino hacia el buen vivir.

Si bien los proyectos de economía social y solidaria que aquí abordamos son algunos de los ejemplos de iniciativas colectivas que están planteando alternativas, no son los únicos. Su elección responde al marco teórico-metodológico utilizado para analizar la emergencia de las alternativas de desarrollo en la frontera desde las epistemologías del sur con el enfoque de la investigaciónacción. Para ello, se tomó como eje metodológico el Diplomado en Economía Social y Solidaria realizado en la Universidad Autónoma de Ciudad Juárez (UACJ) entre mayo y noviembre de 2016.

Entonces el diplomado se convierte en el eje transversal que nos permite reflexionar y aprender, a partir del acercamiento con iniciativas que ya venían trabajando la economía social y solidaria, así como también impulsar nuevos proyectos colectivos. Además, el acercamiento y la colaboración que hemos tenido, nos ha permitido conocer de cerca su filosofía, su trabajo y su organización. Por lo tanto, nuestra investigación se enmarca en las teorías de retaguardia, puesto que no es un análisis frío y objetivo, sino un trabajo que acompaña muy de cerca la labor transformadora del movimiento de economía social y solidaria (Santos, 2010). Los datos de fuentes de información primarias datan de mayo de 2016 a marzo de 2018 y se produjeron a partir de la observación participante y entrevistas semiestructuradas a profundidad realizadas a participantes de los casos de estudio.

Una aproximación a estas experiencias nos muestra las oportunidades, así como los retos y dificultades que se presentan cuando se construye a contracorriente del sistema hegemónico. Su documentación nos permite visibilizar y comprender la forma 
en la que en nuestra región se está construyendo un movimiento de economía social y solidaria como una red de acciones colectivas que avanzan en la construcción del buen vivir, así como concebir las posibilidades para definir los principios de acción en la búsqueda del bien común en la frontera entre México y Estados Unidos.

Para ello, el presente ensayo se estructuró en tres apartados. El primero consiste en el apartado teórico donde se aborda la conceptualización de la economía social y solidaria, así como la diferenciación entre ambas perspectivas. El segundo apartado estriba en la descripción de las experiencias, así como el análisis de las oportunidades y los retos que se presentan para constituirse como alternativas de desarrollo local y regional. Finalmente, en las conclusiones se perfilan líneas de acción y recomendaciones para fortalecer la red del movimiento de economía social y solidaria en la frontera entre México y Estados Unidos.

\section{APUNTES EN TORNO A LAS PRÁCTICAS DE TRANSFORMACIÓN SOCIAL: LA ECONOMÍA SOCIAL Y SOLIDARIA}

Aunque teóricamente las prácticas de economía alternativa han sido llamadas con nombres tan distintos como economía social, economía social y solidaria, economía del trabajo, economía de la solidaridad, economía popular y socioeconomía de la solidaridad, autores como Coraggio, Guerra y Razeto están de acuerdo en que los proyectos tienen implicaciones y formas tan diversas que lo importante son las experiencias colectivas de la transformación social. Para los fines del presente artículo, entendemos la diferencia entre economía social y economía solidaria a partir de la postura política implícita en las prácticas de transformación, no solo de la economía sino también de las relaciones sociales. Es importante aclararlo debido a que, en el caso mexicano, si bien la economía solidaria tiene una amplia trayectoria que se remonta a los pueblos originarios, actualmente el Estado mexicano impulsa políticas públicas más cercanas a la economía social que a la economía solidaria. 
Además, la diferenciación nos permitirá comprender las experiencias que se están realizando en la frontera entre México y Estados Unidos. Aunque ambos conceptos, la economía social y la economía solidaria, tienen sus raíces en movimientos como el mutualismo y el cooperativismo europeo del siglo xIX, se diferencian por tratarse de discusiones y prácticas en contextos muy distintos. Mientras que la economía social se ha desarrollado principalmente en países europeos como Francia, España, Holanda y Alemania, cuando hablamos de economía solidaria nos referimos a prácticas económicas latinoamericanas. Para autores como Mutuberría (2008), la discusión en torno a la economía social y la economía solidaria está en relación con la perspectiva de los países centrales, para la primera; y los países de la periferia, para la segunda.

La economía social "se refiere a las actividades económicas de una sociedad de personas que buscan la democracia económica asociada a la utilidad social" (Defourny, 2009, p. 163). Y al respecto añade que existen dos maneras de describir la economía social: primero, por sus formas jurídicas o institucionales, y segundo, por los rasgos que comparten las organizaciones. En este sentido, cuando hablamos de economía social nos referimos a cooperativas, mutuales y fundaciones que llevan a cabo sus actividades desde los siguientes principios: "a) la finalidad de servicio de los miembros o a la colectividad; b) la primacía de las personas sobre el capital; c) el funcionamiento democrático, y d) la autonomía en la gestión respecto a los poderes públicos" (Guerra, 2014, p. 48).

Las organizaciones que conforman la red del movimiento de economía social afirman que su finalidad es "proveer de manera sostenible las bases materiales para el desarrollo personal, social y ambiental del ser humano" (Red de Redes de Economía Alternativa y Solidaria, 2011). Para ello, realizaron la carta de principios donde expresan los seis valores que deberán regir las prácticas económicas: equidad, trabajo, sostenibilidad ambiental, cooperación, sin fines lucrativos y compromiso con el entorno.

Para el caso mexicano, el Instituto Nacional de la Economía Social (Inaes) define la economía social como el 
conjunto de las actividades económicas y empresariales que [...] llevan a cabo aquellas entidades que persiguen bien el interés colectivo de sus integrantes, bien el interés general económico o social, o ambos. Integra a las mutualidades, a las cooperativas, las asociaciones y a las fundaciones (Inaes, 2014).

Si bien en México muchas de las veces se utilizan como conceptos similares -incluso se habla de economía social y solidaria-, es importante aclarar que la diferencia no solo está en el discurso, sino en las mismas prácticas como acción contracultural ante las lógicas del sistema capitalista.

La economía solidaria, como concepto, es relativamente nuevo, el cual fue acuñado en América Latina a inicios de los años ochenta por Luis Razeto (1997; 2016), quien la define como una búsqueda teórica y práctica de formas alternativas de hacer economía, basadas en la solidaridad y el trabajo. Y al respecto agrega que se trata de un nuevo modo de hacer economía, una nueva racionalidad económica que busca que la solidaridad se introduzca en la economía misma, operando en las diversas fases del ciclo económico: producir con solidaridad, distribuir con solidaridad, consumir con solidaridad, acumular y desarrollar con solidaridad (Razeto, 1997).

Así, cuando hablamos de economía solidaria nos referimos a experiencias más amplias que basan sus prácticas económicas en lazos de reciprocidad que van desde cooperativas, colectivos, asociaciones de trabajadores, comedores, redes de trueque y finanzas alternativas, pero que tienen en común "la primacía de la solidaridad sobre el interés individual y la ganancia material, reflejada en la socialización de los recursos productivos y la adopción de criterios igualitarios" (Laville y Gaiger, 2009, p. 170). Nos referimos también a un modo especial y distinto de hacer economía, que por sus características propias consideramos alternativas respecto de los modos capitalista y estatista predominantes en los mercados determinados (Guerra, 2014) y que, además, permiten una mayor integración social. 
Vemos, pues, que la economía solidaria adquiere características más radicales que la economía social y, por lo general, con un discurso marcadamente más político (Guerra, 2014). Nuestra postura parte de una diferenciación radical entre la economía social y la economía solidaria en el sentido de la observación de Marañón (2016), quien señala que se ha considerado como economía solidaria "las experiencias que no obtienen ingresos a través del mercado” (p. 26), aunado a la idea de Razeto (1997), quien indica que la economía solidaria tiene una orientación crítica y transformadora respecto de las grandes estructuras y los modos de organización y de acción que caracterizan la economía contemporánea. Mientras que la economía social es un movimiento de resistencia de la población marginada de los procesos laborales y productivos donde crean su propia empresa sin la necesidad de cuestionar el sistema capitalista, ya que utilizan sus medios como una forma de resistencia no radical, compatible con el sistema, buscando resolver y aminorar las problemáticas sociales que el capitalismo ha generado, tal como el desempleo, la desigualdad y la exclusión, entre otros. Por su parte, la economía solidaria es un paradigma poscapitalista al ser una forma de organización anticapitalista desde la cual podemos crear otra economía.

A continuación presentamos una reflexión en torno a cuatro proyectos que actualmente se están promoviendo en el norte de México y el sur de Estados Unidos: la cooperativa Mujeres de Esperanza y Fe y la cooperativa Servicio Verde, que impulsan proyectos de economía social; mientras que el colectivo Chopeke y los bancos de tiempo desarrollan proyectos de economía solidaria. Para ello, seguiremos las recomendaciones de Marañón (2016), quien señala tres cuestiones que debemos observar en el análisis crítico de las experiencias de la economía alternativa. En primer lugar, es importante observar al interior de las organizaciones el tipo de relaciones sociales que se tejen entre los miembros con la finalidad de determinar el tipo de racionalidad que impera. ${ }^{2}$

2 Al respecto, Boris Marañón (2016) indica que debemos preguntarnos si existen relaciones de explotación y/o dominación, y cuál es el espacio de la reciprocidad, la igualdad, la comunidad y la sustentabilidad. 
En segundo lugar debemos contar con una definición operativa donde se incluyan los criterios que transforman las relaciones sociales. En tercer lugar, apunta a preguntarnos sobre la relación de las iniciativas con el sistema económico capitalista y el mercado. ${ }^{3}$

\section{LA EMERGENCIA DE ALTERNATIVAS DE DESARROLLO LOCAL Y REGIONAL}

Actualmente en México, el movimiento de economía social y economía solidaria está creciendo. Sus prácticas están generando alternativas y en las universidades del país se empiezan a desarrollar cursos, seminarios, encuentros y programas donde se discuten y analizan las economías alternativas.

En el caso de Ciudad Juárez, urbe fronteriza, industrial y con una población en su mayoría migrante, la economía social y la economía solidaria merecen un estudio adicional debido a su condición fronteriza. Ciudad Juárez y El Paso reúnen a una población de dos millones y medio de personas, formando la zona metropolitana binacional más grande a lo largo de la frontera MéxicoEstados Unidos. ${ }^{4}$ Por su dinámica industrial y de servicios ligada a la inversión extranjera, el crecimiento poblacional ha repercutido sobre las oportunidades laborales y productivas, lo cual se manifiesta en el ingreso de la población, así como en la disposición y cobertura de los servicios públicos.

Ciudad Juárez ha sufrido las consecuencias de la crisis económica mundial. Entre 2008 y 2009 se perdieron poco más de ochenta mil plazas laborales y $56.5 \%$ de quienes cuentan con un empleo formal reciben salarios insuficientes, puesto que van de uno a tres salarios mínimos (Plan Estratégico de Juárez, A. C., 2017). Como indica el Informe 2015 Así estamos Juárez, 51.5 \% de los juarenses expresan que no les alcanza el ingreso familiar para vivir de manera adecuada: este porcentaje crece al $60 \%$ en el caso de

3 Marañón (2016) plantea la pregunta: ¿en realidad son parte de otro mundo si al mismo tiempo tienen que producir, vender y lograr sus ingresos, parcial o totalmente, a través de relaciones mercantiles?

4 Información obtenida de www.chihuahuamexico.com contrastada con datos del Censo de Población y Vivienda 2015, realizado por el Inegi, y el Diagnóstico territorial de Ciudad Juárez, llevado a cabo por el Plan Estratégico de Juárez, A. C. 
la población mayor de sesenta años (Plan Estratégico de Juárez, A. C., 2015). Y según la Encuesta de Percepción Ciudadana 2016 (Plan Estratégico de Juárez, A. C., 2016), la ciudadanía juarense considera que su situación económica ha empeorado respecto de los años anteriores.

Las condiciones de pobreza en las que vive una gran parte de la población, aunado a la violencia extrema cotidiana y la desaparición sistemática de mujeres, han debilitado el capital social de las comunidades. La industria maquiladora intensifica la polarización social y espacial al configurar y dividir el espacio urbano en dos grandes zonas: el poniente y el oriente. El poniente, lugar de asentamientos irregulares donde se asentó la mayoría de la población migrante, presenta graves problemas sociales, económicos y ambientales, además de los mayores déficits de infraestructura y equipamiento de la ciudad (IMIP, 2002), lo que ha llevado a que autores como Bass, Pérez y Ramírez (2010) hablen de una urbanización marginal resultado de la ocupación del suelo espontánea y carente de servicios públicos. Por otra parte, la zona contrasta con el oriente de Ciudad Juárez, donde se ubica la mayor infraestructura y equipamiento urbanos. Aunado a ello, las actuales condiciones de empleo se caracterizan por ser sumamente precarias, ya que explotan, agotan y desechan a los seres humanos a favor de la productividad y los bajos costos (Jusidman y Almada, 2008).

La exploración de la economía solidaria cobra cada día más relevancia cuando hablamos de aspirar a una sociedad más justa, equitativa y solidaria frente a un desalentador panorama donde $37 \%$ de la población de Ciudad Juárez vive en pobreza y de los cuales poco más de 62000 personas se encuentran en pobreza extrema con un ingreso menor al de la canasta alimentaria y con las necesidades de salud, educación, seguridad social, vivienda e infraestructura social básica no cubiertas (El Diario de Juárez, 2013).

Sin embargo, en el contexto de la región fronteriza Ciudad Juárez-El Paso están surgiendo saberes, prácticas y actores de transformación social que proponen otras formas de hacer economía para satisfacer sus necesidades, así como para el desarrollo de las comunidades fronterizas. Con la idea de convertir a la universidad 
en un espacio de reflexión, encuentro e incubación, tanto de la economía social como de la solidaria, se realizó el Diplomado en Economía Social y Solidaria impartido entre mayo y noviembre de 2016 en la UACJ. Dicho programa tuvo como objetivo principal estimular el debate revelando alternativas, buscando disminuir las desigualdades sociales, a través de reunir las capacidades y recursos de los participantes, y formando sinergias que revitalicen las comunidades y fortalezcan el desarrollo local y regional, a partir de generar capital social a escala local, poniendo énfasis en las relaciones de solidaridad y confianza, y en la participación en la sociedad.

Para lograrlo se buscó comprender las dimensiones teóricas y prácticas de la economía social y la economía solidaria. Primero, teniendo claridad conceptual en torno al tema y reflexionando a propósito de los principios y valores en los que se basan las economías alternativas, así como el marco legal en el que se insertan. Y segundo, conocer las iniciativas que se están desarrollando en la región, para posteriormente desarrollar propuestas propias derivadas del aprendizaje colectivo. De esta manera, fue que dentro del marco del diplomado se realizó el acercamiento a iniciativas como la cooperativa Mujeres de Esperanza y Fe, colectivo Chopeke y el banco de tiempo DACU, y se creó la cooperativa Servicio Verde, la moneda alternativa Nochari, la comunidad de talentos y, posteriormente, el banco de tiempo de Ciudad Juárez.

\section{CASOS DE PRÁCTICAS DE LA ECONOMÍA SOCIAL}

A continuación presentamos la experiencia de la cooperativa $\mathrm{Mu}$ jeres de Esperanza y Fe y la cooperativa Servicio Verde, que han impulsado proyectos de economía social. La primera iniciativa desde hace veinte años y la segunda, se forma por estudiantes del Diplomado en Economía Social y Solidaria en 2017.

\section{COOPERATIVA MUJERES DE ESPERANZA Y FE}

La cooperativa Mujeres de Esperanza y Fe se encuentra en el centro Santa Catalina, ubicado en la colonia Pánfilo Natera en el po- 
niente de Ciudad Juárez. El centro Santa Catalina fue fundado en 1996 por Donna Kustusch y Eleanor Stech, dos monjas dominicas. La cooperativa se inició en 1997 con el apoyo de Elizabeth Pepe y Roseann Schlitt, quienes tenían experiencia en el movimiento cooperativista en la República Dominicana.

Las mujeres de la colonia tienen poco más de veinte años trabajando bajo los principios de solidaridad, cooperación, equidad y compromiso con su entorno. Ellas iniciaron su cooperativa haciendo flores de papel en un cuarto atrás de la iglesia. En un inicio eran treinta mujeres -en la cooperativa- y actualmente participan alrededor de diecinueve, diez de las cuales son parte de la mesa directiva que se renueva anualmente. Ellas mismas, y de manera colectiva, establecieron sus horarios de trabajo, acordando que sería de martes a jueves de nueve de la mañana a doce del mediodía. Los viernes son días especiales, por lo que cada viernes del mes se destina a distintas actividades. El primer viernes de cada mes lo dedican a la oración; el segundo, a estudiar; el tercero, a misa; y el cuarto, a talleres de capacitación. Asimismo, establecieron los requisitos para formar parte de la cooperativa, acordando que las interesadas tendrán tres meses de prueba; deberán acudir a todas las actividades de los viernes; y no deben faltar ni llegar tarde, y acudir a misa.

Todas las mujeres trabajan de manera equitativa en la elaboración de productos de costura como manteles y rebozos que venden en Estados Unidos. Los gastos y las ganancias los distribuyen en partes iguales. Han creado un fondo de ahorro y préstamo, y cuando alguna de ellas se enferma, todas trabajan para que siga recibiendo su dinero mensualmente hasta por tres meses. A los seis meses entregan la mitad y luego de seis meses, les es imposible continuar, por lo que el apoyo económico se termina. En promedio sus ingresos son de $\$ 160$ dólares $^{5}$ al mes. Aunque para muchas de ellas es su única fuente de ingresos, para otras no es suficiente, por lo que se ven obligadas a buscar empleo en la industria maquiladora.

5 Aproximadamente $\$ 2800$ pesos con el tipo de cambio actual, lo que significa un ingreso de alrededor de $\$ 700$ pesos semanales. 
La experiencia de veinte años donde la cooperativa se ha convertido en un espacio de conocimiento, apoyo y trabajo colectivo que ha brindado no solo ingresos extra a quienes ahí participan, sino que, además, ha fortalecido el capital social en la colonia.

En el marco del Diplomado en Economía Social y Solidaria reflexionamos de manera colectiva sobre el aprendizaje que obtuvimos de su experiencia y se redactó una retroalimentación que, posteriormente, se hizo llegar a la cooperativa. Los retos que a continuación presento, se basan en gran parte en dichas observaciones.

Si bien han logrado generar ingresos con la venta de sus productos en Estados Unidos, no ha sido suficiente para un buen vivir, ya que muchas de ellas aún tienen que vender su fuerza de trabajo a la industria maquiladora.

Al dedicarse la mayor parte del tiempo a la producción, no se han ocupado de la promoción y/o distribución de sus productos más allá de la red de iglesias con las que el centro Santa Catalina colabora. Por lo tanto, son poco conocidas en el ámbito local y regional. Destinar una comisión de la cooperativa a la promoción y creación de alianzas con otros productores y distribuidores, les puede ayudar a tener mayor presencia en el mercado local y regional.

Si bien el empuje y apoyo que les ha dado la iglesia ha sido muy importante para la existencia de la cooperativa, el no contar con un espacio propio les ha generado problemas, desencuentros y rupturas al interior de la organización. El espacio propio puede ser una meta a alcanzar para lograr la autonomía de la cooperativa.

Otro eje que han descuidado al dedicarse casi exclusivamente a la producción, ha sido la educación en economía social. Toda cooperativa debe dedicar parte de su organización a la formación y capacitación. Ello implica disponer de tiempo y recursos para actualizarse en métodos y herramientas que les permitan mejorar su trabajo no solo en la producción, sino también en la distribución, en la gestión de la cooperativa como las cuestiones legales y administrativas, en la relación con los proveedores y clientes, así como en la constante reflexión de los principios de la economía social.

Actualmente, la cooperativa no tiene acceso al IMsS o al Seguro Popular. Si bien son solidarias con las compañeras y si alguna de 
ellas se enferma, la cooperativa la apoya, no es suficiente hacerlo solamente por seis meses. ¿Qué pasa con los accidentes de trabajo o con las enfermedades crónicas? La solidaridad no debe tener límites. La cooperativa debe considerar una caja de ahorros no solo para préstamos, sino también para servicios médicos, para casos de defunción e, incluso, para el retiro.

\section{COOPERATIVA SERVICIO VERDE}

Aunque de los tres planes de cooperativa creados en el Diplomado en Economía Social y Solidaria, todos tenían intenciones de ser ejecutados, esto no fue así. Las propuestas consistían en una cooperativa distribuidora de artesanías; una cooperativa de educación titulada "Universidad Popular"; y otra de consumo de productos del campo chihuahuense titulada "Del Campo a su Mesa". La primera estuvo integrada por un grupo de base apoyado por la Fundación del Empresariado Chihuahuense (Fechac) con recursos económicos y talleres de capacitación. Debido a que ya habían trabajado juntos en proyectos en sus colonias, fue el grupo con mayor cohesión y que compartían identidad e intereses, por lo que para ellos no fue complicado desarrollar y continuar con el proyecto. Si bien no se conformaron como una cooperativa, a diferencia de lo que ocurrió con los demás grupos, siguen produciendo y distribuyendo sus productos, y colaborando entre sí. Una vez terminado el diplomado, quienes elaboraron el proyecto de la "Universidad Popular" se desintegraron y el proyecto se quedó solamente en papel. El tercer grupo que propuso la cooperativa "Del Campo a su Mesa”, a poco más de un año de trabajar juntos, ha presentado las situaciones más complejas e interesantes. Debido a los retos y complicaciones para llevar a cabo el proyecto original, lo transformaron completamente hasta convertirlo en la cooperativa Servicio Verde, que ofrece un servicio profesional de lavado de autos y muebles en seco.

Si bien el diplomado aportó 144 horas de formación teórica, así como herramientas para la creación y fortalecimiento de las prácticas colectivas, luego de poco más de un año aún existen 
dificultades para efectuarlas debido a la complejidad del sistema económico en el que se insertan. En este sentido, quienes participaron en el diplomado han realizado actividades que en el discurso son economía social, pero que en la práctica aún están muy alejadas de serlo.

A pesar de haber terminado satisfactoriamente el diplomado y de haber obtenido las bases teóricas y las herramientas necesarias para iniciar con los proyectos, el grupo no logró poner énfasis en el factor $\mathrm{C}$ ni en los principios de la economía social, sino que han estado más interesados en realizar una empresa que satisfaga solamente sus necesidades monetarias. El grupo impulsor de la cooperativa originalmente estaba conformado por seis miembros, tres de los cuales buscaban impulsar un proyecto de economía social y otros tres estaban interesados en fortalecer la economía solidaria. Luego de variadas discusiones al respecto y a la imposición de un nuevo proyecto de economía social por parte de quienes tenían la necesidad de insertarse en el mercado con un proyecto de autoempleo, aunado a la poca disponibilidad de diálogo, se debilitó el grupo hasta el grado de que la mitad de sus miembros decidieron abandonarlo. Actualmente, y luego de cerca de año y medio de trabajo, el proyecto no ha logrado dar el salto abismal de la teoría a la práctica ni ha logrado consolidarse como un proyecto que resuelva las necesidades de sus miembros.

\section{CASOS DE PRÁCTICAS DE LA ECONOMÍA SOLIDARIA}

En cuanto a los proyectos de economía solidaria, a continuación presentamos la experiencia del colectivo Chopeke y el banco de tiempo DACU, con quienes tuvimos un acercamiento en el marco del Diplomado en Economía Social y Solidaria, así como las iniciativas creadas posteriormente por los estudiantes, como es el caso de la moneda social Nochari y el banco de tiempo de Ciudad Juárez. 


\section{COLECTIVO CHOPEKE}

Chopeke $^{6}$ es una comunidad de jóvenes católicos que se reúnen en el año de 2014 y ante la pregunta: ¿cómo respondemos a la necesidad social?, deciden enfrentar la falta de vivienda digna en Ciudad Juárez a través de la arquitectura colaborativa. Con materiales como tierra, piedra de escombro y pajas de trigo del valle de Juárez en tan solo tres años han realizado seis construcciones. La primera fue una vivienda de $25 \mathrm{~m}^{2}$ en el kilómetro 33 de la carretera a Casas Grandes, donde habita una comunidad rarámuri. Actualmente trabajan en la ampliación de una casa y lo que será un centro de salud alternativa en Lomas de Poleo. Su trabajo fue reconocido en la Bienal de Venecia por la forma en la que enfrentan los desafíos de construcción con responsabilidad social y creatividad, mejorando la condición del entorno construido y, con ello, la calidad de vida de sus habitantes (Secretaría de Cultura, 2016).

El caso de la capilla Santa Luisa de Marillac en la colonia Adolfo López Mateos es muestra de lo que la solidaridad y el trabajo colectivo pueden lograr. Cuando el seminarista Paul Pérez, miembro del colectivo Chopeke, es enviado al lugar, se da cuenta de que la comunidad no está satisfecha con las condiciones de su capilla y que, incluso, las personas ya no acuden porque no se sienten cómodas en el lugar. Todo empezó en enero de 2015 con dos preguntas: ¿qué soñamos y qué necesitamos? El proyecto requería \$150 000 pesos solo en materiales, ya que todo fue autogestivo y ninguno de los participantes recibió un pago, por lo que todo el trabajo se aportó solidariamente. Con eventos en la misma colonia como ventas de comida, fiestas y verbenas populares, rápidamente lograron juntar el capital necesario. En junio se reunió la comunidad y con sus propias manos derrumbaron la vieja capilla y comenzaron la construcción de lo que sería su nuevo santuario: un templo construido con la técnica del bahareque con acabado de mortero ligero y encalado natural. El sueño de la comunidad hecho realidad colectivamente, construido con su

6 Ocote en rarámuri. 
esfuerzo y la participación de hombres, mujeres, niños, jóvenes y ancianos reunidos para transformar su espacio.

El trabajo del colectivo Chopeke es una muestra de lo que una comunidad organizada bajo los principios de solidaridad puede hacer cuando asocia su trabajo para avanzar hacia un sueño común. En la región fronteriza se convierten en el ejemplo de que otro mundo es posible si nos organizamos y ponemos en marcha valores como la cooperación, la ayuda mutua, la responsabilidad con el entorno, la sustentabilidad ambiental, la equidad y el trabajo colectivo.

El impacto que ha tenido el colectivo Chopeke, se debe a la manera en la que se han organizado, al valor que han dado a la formación del capital social construyendo lazos de confianza y a la discusión colectiva de la ética comunitaria. En este sentido, tanto los estudiantes como los profesionales de la arquitectura y la ingeniería, al surgir de una comunidad eclesiástica que comparte una identidad y los valores de la doctrina social de la Iglesia, entienden la solidaridad como un deber ser. Comparten una filosofía que busca la transformación de la realidad y, como ellos mismos lo mencionan, desde la Iglesia están apostando por el cambio de estructura, pasar de ver y juzgar al actuar, superando el asistencialismo y la promoción en la búsqueda del buen vivir.

Sin embargo, el colectivo Chopeke también se enfrenta a grandes retos. Cuando se preguntan: ¿qué podemos hacer para cambiar el mundo?, el mayor reto es mantener y extender la esperanza en un ambiente de violencia extrema y de prácticas hegemónicas que, a pasos agigantados, destruyen el ambiente y construyen subjetividades donde la mayoría naturaliza la explotación, la pobreza, el afán de lucro, el individualismo y el egoísmo, haciéndonos creer que otras formas de vida son imposibles. El reto para el colectivo Chopeke es continuar soñando, amando y construyendo en comunidad, para demostrar que podemos superar las actuales condiciones de inequidad, marginación, exclusión y deterioro ambiental.

Además, el colectivo invierte recursos humanos, tecnológicos y financieros de manera voluntaria que solo ha encontrado en personas de la comunidad católica de la frontera. El reto iría en dos 
sentidos: la sostenibilidad del proyecto y las redes de colaboración. Primero, lograr la sostenibilidad económica del proyecto. Si bien ya practican la economía solidaria y han logrado empoderar a las comunidades, los costos que el colectivo asume pueden debilitar a los participantes, o bien, la ausencia de alguno puede desequilibrar el proyecto. Por ello, es importante promover la participación de las personas más necesitadas de manera horizontal, para que sean ellas mismas quienes resuelvan sus necesidades. Segundo, el reto sería mover voluntades de otra naturaleza, lo que implica ampliar las redes de colaboración con otros colectivos, organizaciones e, incluso, con las instituciones promotoras de vivienda.

\section{BANCOS DE TIEMPO Y MONEDAS SOCIALES}

Siguiendo con las iniciativas de economía solidaria ahora presentaremos los proyectos que buscan desmercantilizar las relaciones sociales; para ello, utilizan las finanzas alternativas, tal es el caso de las monedas sociales y los bancos de tiempo.

Las monedas alternativas se definen como instrumentos monetarios derivados de colectividades de personas con vocación no comercial y se caracterizan por nacer en grupos sociales organizados, con el fin de intercambiar bienes y servicios sobre la base del trueque y de valores sociales alternativos (Guerra, 2014). Con esta idea, estudiantes del diplomado en Ciudad Juárez, inspirados en el Túmin, ${ }^{7}$ forman una comunidad de talentos para intercambiar sus conocimientos y servicios, y para ello crean el Nochari. ${ }^{8}$ Aunque en la comunidad se inscribieron cerca de veinte personas, se realizaron varias reuniones e intercambios y luego del acercamiento con el banco de tiempo DACU, se replantearon las formas de realizar los intercambios.

7 Túmin en totonaca significa dinero. Es una moneda social creada en 2010 en la comunidad El Espinal, Veracruz. Para más información recomendamos: Junta de Buen Gobierno (2014). Aceptamos Túmin: mercado alternativo, economía solidaria y autogestión. Disponible en: http://www.uv.mx/uvi/files/2014/12/Libro-Aceptamos-Tuminversion-final.pdf

8 Significa trabajo en rarámuri. 
DACU es un proyecto ubicado en Las Cruces, Nuevo México. Tiene como objetivo fortalecer la capacidad de las comunidades para desarrollar e implementar políticas y soluciones comunitarias para mejorar las condiciones sociales, económicas y ambientales, trabajando para promover la equidad en salud en el condado de Doña Ana, al abordar las inequidades institucionales relacionadas con la geografía, la clase, el género y la raza (DACU, 2017). Desde 2015 realizan un banco de tiempo en el que actualmente participan 231 miembros, quienes han intercambiado 1613 horas en servicios. ${ }^{9}$

Los bancos de tiempo son una

propuesta para transformar la experiencia colectiva del tiempo, basada en sistemas de intercambio no monetarios entre las personas y sus comunidades, promoviendo y valorando la acción colaborativa motivada desde los afectos y desde la abundancia que surge de compartir el tiempo (Coordinación Colaborativa de Rotación de Tiempo, 2016).

En la reunión de los organizadores de la comunidad de talentos en Ciudad Juárez y del banco de tiempo DACu en el marco del Diplomado en Economía Social y Solidaria, se planteó la posibilidad de unir las iniciativas con la intención de crear un banco de tiempo transfronterizo donde participen los habitantes de Ciudad Juárez, El Paso y Las Cruces. Desde esa reunión ocurrida en septiembre de 2016, se han intercambiado servicios como transporte y clases de inglés y español, y se han efectuado dos visitas recíprocas. En mayo de 2017 miembros del banco de tiempo acudieron a visitar a los miembros de Las Cruces, donde compartieron sus intereses y visiones sobre la frontera; finalmente, hablaron sobre sus expectativas de colaboración con el enfoque de la economía solidaria. La comunidad de talentos y el banco de tiempo, se articulan y reúnen a personas de tres ciudades que históricamente han sido separadas por razones geográficas, políticas

9 Información obtenida de la plataforma www.hOurworld.org, la red internacional de bancos de tiempo. 
y económicas. Con esta idea, en enero de 2018, se crea el banco de tiempo de Ciudad Juárez que ahora nos reúne sin intenciones lucrativas, más bien solidarias, generando capital social para crear comunidades que superen las limitaciones que impone la frontera.

El banco de tiempo DACU ha encontrado la forma de crear una red que reúna a las comunidades de Las Cruces para realizar intercambios gratuitos. Su ubicación en la región fronteriza, le da la oportunidad de ampliar las redes de colaboración, incluyendo ciudades como El Paso y Ciudad Juárez.

Han logrado crear redes de colaboración con las instituciones locales de educación, de seguridad pública y de salud, para incluir en la red de intercambios a personas marginadas y excluidas socialmente.

Tanto el banco de tiempo de DACU como el de Ciudad Juárez dan valor al tiempo de las personas de manera igualitaria y generan capital social, la base de todo proyecto de economía solidaria. Además, se reúnen regularmente para compartir experiencias y conocimientos, impulsando la creación de nuevos proyectos.

La ubicación geográfica de Las Cruces y Ciudad Juárez favorece a los bancos de tiempo, pues su condición de frontera potencia los intercambios en tres ciudades. Si bien ya se inició el banco de tiempo transfronterizo, el reto es mantenerlo y que siga creciendo, donde se intercambien servicios gratuitos a ambos lados de la frontera.

De ahí se desprenden dos retos relacionados con la economía fronteriza: las desigualdades económicas, y la oferta y la demanda de los servicios. En el caso de las desigualdades económicas entre las comunidades de ambos lados de la frontera, se pueden crear prácticas verticales con una visión asistencialista. En este sentido, el reto sería generar formas de organización horizontales basadas en la cooperación y la solidaridad comprendiendo que las necesidades son distintas en ambos lados de la frontera, pero superando la dependencia que caracteriza al sistema económico. En cuanto a la oferta y la demanda de los servicios, el reto es incluir a aquellos que son de primera necesidad. Si bien es importante el intercambio del tiempo en sí mismo, ya que es lo que permite crear co- 
munidades con cohesión social y redefinir el valor del tiempo, se debe ampliar la red e incluir el intercambio de servicios que nos permitan prescindir del dinero para resolver las necesidades en relación con oficios que necesitamos en la vida diaria. Actualmente, el banco de tiempo se caracteriza por ofrecer servicios educativos, de acompañamiento y ocio como lecturas, apoyo en transporte y organización de eventos. Si se incluyen servicios como mecánica, plomería, servicios médicos, psicológicos, jurídicos, contabilidad y estancias infantiles, entre otros, el proyecto tendría mayor impacto. Ello lo radicalizaría, permitiendo que poco a poco disminuyan las necesidades en las que utilizamos el dinero.

\section{CONCLUSIONES}

Las experiencias colectivas de economía alternativa en la frontera México-Estados Unidos muestran que, si bien buscan satisfacer necesidades sociales específicas, parten del desconocimiento de la economía social y la economía solidaria, puesto que, a excepción de los impulsores del banco de tiempo DACU y el de Ciudad Juárez, no dedican tiempo al estudio de la economía y/o de los valores y principios que deberán guiar las prácticas.

Es necesaria la construcción y promoción de programas de estudio para fortalecer el conocimiento de la economía alternativa. Es aquí donde se brindarán las herramientas que permitan fortalecer los proyectos que actualmente se realizan, así como la creación de nuevas iniciativas a partir de la sensibilización de aquellas personas que aún no participan o no conocen el movimiento de la economía solidaria. Y, además de la parte técnica, es necesario crear foros de discusión donde coincidan las cooperativas que forman parte del movimiento con los estudiantes e investigadores con un doble objetivo: por una parte, con la intención de aprender de su experiencia, y por otra, para la creación de redes de colaboración que solo son posibles cuando se crea el espacio para que se sienten a dialogar. Y, finalmente, la cuestión ético-filosófica de la economía social y la economía solidaria. Es importante, también, reflexionar en torno a sus principios y en la forma en la 
que se trasladan a la vida cotidiana. Es necesario promover una constante reflexión en torno a los valores y la ética comunitaria, cuestionando aquellas prácticas que se dicen de economía social y solidaria, pero que no lo son, y en su lugar reproducen las desigualdades. Y hay que estar atentos, porque frente al modelo capitalista las prácticas alternativas quedan invisibilizadas e, incluso, absorbidas.

En contraste, existen iniciativas y políticas públicas que se están apropiando del discurso de la economía social y la economía solidaria, pero que nada tienen que ver con ellas y que lo único que consiguen es fortalecer la subjetividad capitalista, a través de modelos de emprendimiento que inducen a los sujetos a la autoexplotación, al endeudamiento y, posteriormente, a la frustración cuando los proyectos no logran sus objetivos. Ello afecta a aquellas iniciativas que sí buscan el bien común, puesto que crea un ambiente de desesperanza y desilusión.

Partiendo de nuestras definiciones teóricas, podemos concluir que las experiencias aquí presentadas todavía no se consolidan como alternativas, puesto que los casos de economía social, como la cooperativa Mujeres de Esperanza y Fe, así como la cooperativa Servicio Verde, si bien tienen como finalidad el servicio a los miembros o a la colectividad, no está claro la primacía de las personas sobre el capital, no existe un funcionamiento democrático ni se ha conseguido la autonomía en la gestión.

Respecto a las experiencias de economía solidaria, en ambos casos, tanto en el colectivo Chopeke como en el banco de tiempo DACU y el de Ciudad Juárez, no se obtienen ingresos a través del mercado y tienen una orientación crítica; su capacidad de transformación de las estructuras todavía no logra empoderar a los participantes desde una perspectiva humanista para convertirse en una praxis desde abajo, donde se estimulen las capacidades de la población para la formación del capital social. Entendemos que solo si se parte del factor humano estimulando la confianza y reciprocidad, con colectivos que compartan identidad y valores, con normas de conducta cívica claras y dispuestos a trabajar de manera colectiva, se podrán impulsar proyectos que hagan frente 
al asistencialismo y clientelismo que caracteriza a los programas sociales federales, estatales y municipales.

En cuanto a las relaciones tejidas al interior de los colectivos presentados, en el caso de aquellos dedicados a las economía social, si bien no son de explotación, sí son de dominación, puesto que las relaciones no son del todo horizontales ni equitativas y en ambos casos se ha demostrado que no son sustentables y que no existe espacio para la reciprocidad. Mientras que en el caso de las experiencias de la economía solidaria, las relaciones que se tejen tampoco son de explotación y/o dominación, y existe espacio para la reciprocidad, la igualdad y la comunidad, lo que está en juego es la sustentabilidad de las iniciativas, puesto que en ninguno de los casos aquí presentados existe un proyecto de gestión amplio que implique la perdurabilidad de la empresa.

Finalmente, hablando de las relaciones al exterior de los colectivos, con el mercado y con el sistema económico capitalista, en ambos casos es el tema más complejo, puesto que las dificultades que el movimiento de la economía social y la economía solidaria enfrenta en nuestra región están en relación con las prácticas económicas hegemónicas y asociadas a su condición de frontera. Impulsar un movimiento de economía social y economía solidaria en el límite geográfico de México y Estados Unidos tiene diversas implicaciones: económica, política y social. La frontera puede ser un factor que favorezca las acciones colectivas, pero también puede complicarlas y desgastarlas. Encontrarnos como comunidades limítrofes puede ser favorable, puesto que aquello que social, política y económicamente compartimos nos permite crear lazos de confianza, a partir de la reflexión sobre esos sistemas de control e instrumentación de las relaciones. Sin embargo, el límite geográfico también rompe la red del movimiento de economía social y economía solidaria, al dificultar la circulación y la comunicación no solo de personas y mercancías, sino también de las ideas, habituándonos a prácticas en las que, por sus implicaciones, es complicado imaginar alternativas para superarlas.

Si aplicamos lo dicho por Boaventura de Sousa (2010) al hablar de las dificultades de la imaginación política, en Ciudad Juárez 
nos encontramos con que es "tan difícil imaginar el fin del capitalismo como que el capitalismo no tenga fin” (p. 27). En la frontera entre México y Estados Unidos vivimos tan preocupados por sobrevivir, y los académicos y los políticos están tan ocupados en desarrollar diagnósticos, análisis, políticas públicas y programas que expliquen o minimicen los costos sociales de la acumulación capitalista que no se cuestiona al sistema en sí mismo. Las prácticas de la economía social y la economía solidaria nos permiten soñar; a pesar de las condiciones estructurales de la ciudad, a pesar de nuestra condición de pobreza y la violencia extrema, es posible la construcción de proyectos que permitan la creación colectiva de una vida digna. Favorece la creación de lazos de confianza y la (re)construcción del capital social en las comunidades. Nos permite creer que construir otro mundo es posible. Pero que si vamos a construirlo otra economía es la condición para lograrlo (Hinkelammert, \& Mora, 2006).

Los proyectos de economía social y economía solidaria, se componen por el capital y sus categorías organizadoras: el trabajo, la tecnología entendida como el saber hacer, la gestión que consiste en la administración de la iniciativa y el factor $\mathrm{C}$ o capital social, el factor humano. La experiencia del movimiento de economía social y economía solidaria muestra que la construcción de la confianza, la reciprocidad, los valores y la identidad compartida son las características que debe tener el capital social para que los proyectos sean exitosos. Si la iniciativa comienza desde abajo con la construcción de la comunidad, será más fácil reunir el capital y la tecnología, así como realizar la gestión de manera colectiva. Muchas veces los proyectos fracasan, porque empiezan con la dotación del capital monetario descuidando el capital social. En este sentido es que van los retos para impulsar un movimiento de economía social y economía solidaria en la frontera. Si bien ya se está demostrando que está aportando alternativas que nos permiten acercarnos al buen vivir, los retos que presentan los podemos agrupar en relación con la parte teórica-conceptual y la parte práctica. 
En cuanto a la cuestión práctica, como hemos mencionado, uno de los retos se encuentra en el salto del discurso a la praxis, así como en la consolidación del capital social. Este es el mayor reto: construir la autonomía colectivamente, de manera horizontal y bajo los principios de la economía social y la economía solidaria. Frente al desolador panorama que se vive en la frontera norte de México, el reto es no perder la esperanza y el primer paso es imaginar y empezar a construir otras formas de vivir en comunidad, para demostrar que es posible y que el movimiento continúe creciendo y rompiendo fronteras.

\section{FUENTES CONSULTADAS}

Bass, S., Pérez, M. E., \& Ramírez, J. (2010). Análisis de las dinámicas de violencia de género y su relación con las condiciones de la vivienda en el poniente de Ciudad Juárez. Ciudad Juárez: Universidad Autónoma de Ciudad Juárez.

Coordinación Colaborativa de Rotación de Tiempo (CoCoRoCLOCK) (2016). Cómo hacer abundante el tiempo. México. Recuperado de http://cocoroclock.tumblr.com/post/154260533309/ como-hacer-abundante-el-tiempo

Defourny, J. (2009). Economía solidaria. En A. Cattani, J. Coraggio, \& J.-L. Laville, Diccionario de la otra economía (1. ${ }^{\text {a }}$ ed.). Buenos Aires: Altamira.

Doña Ana Communities United (2017). Timebank. Las Cruces: Doña Ana Communities United. Recuperado de http://www. da-cu.org/join/

El Diario de Juárez (2013). Tiene Juárez 432 mil pobres y 62 mil en pobreza extrema. Recuperado el 28 de diciembre de 2015, de http://diario.mx/Local/2013-04-12_30a84ffc/tiene-juarez-432-milpobres-y-62-mil-en-pobreza-extrema/

Guerra, P. (2014). Socioeconomía de la solidaridad: una teoría para dar cuenta de las experiencias sociales y económicas alternativas. Bogotá: Ediciones Universidad Cooperativa de Colombia. 
Hinkelammert, F., \& Mora, J. (2006). Hacia una economía para la vida: preludio a una reconstrucción de la economía. Costa Rica: Editorial Tecnológica de Costa Rica.

Instituto Municipal de Investigación y Planeación (IMIP) (2002). Plan de Desarrollo Urbano de Ciudad Juárez. Ciudad Juárez: Instituto Municipal de Investigación y Planeación.

Instituto Nacional de la Economía Social (Inaes) (2014). ABC de la economía social e Inaes. México. Recuperado de https://www. gob.mx/cms/uploads/.../ABC_de_la_Econom_a_Social_e_ INAES.pdf

Instituto Nacional de Estadística y Geografía (Inegi) (2015). Producto interno bruto. Recuperado el 28 de diciembre de 2015, de http://www.inegi.org.mx/est/contenidos/proyectos/cn/pibt/ Jusidman, C., \& Almada, H. (2008). La realidad social de Ciudad Juárez. Ciudad Juárez: Universidad Autónoma de Ciudad Juárez. Laville, J., \& Gaiger, L. (2009). Economía solidaria. En A. Cattani, J. Coraggio, \& J.-L. Lavilla, Diccionario de la otra economía (1. ${ }^{a}$ ed.). Buenos Aires: Altamira.

Marañón, B. (2016). Políticas para la solidaridad económica y el buen vivir en México. México: Universidad Nacional Autónoma de México/Instituto de Investigaciones Económicas.

Mutuberría, V. (2008). El debate en torno a la economía social. Discusiones fundamentales desde la perspectiva de los países centrales y la perspectiva de los países de la periferia. Revista Idelcoop, 35(183), 22-36.

Padilla, H., Olivas, C., \& Alvarado, L. (2014). Ciudad Juárez y la necesidad de política: de la ciudad real a la ideal. Ciudad Juárez: Universidad Autónoma de Ciudad Juárez.

Plan Estratégico de Juárez, A. C. (2015). Informe 2015 Así estamos Juárez. Ciudad Juárez: Plan Estratégico de Juárez. Recuperado de http://www.planjuarez.org/images/docs/informe_aej_2015.pdf ---- (2016). Encuesta de Percepción Ciudadana 2016. Ciudad Juárez: Plan Estratégico de Juárez.

(2017). Pobreza en Juárez: deficiencia en la atención a zonas vulnerables. Ciudad Juárez: Plan Estratégico de Juárez. 
Razeto, L. (1997). Los caminos de la economía de solidaridad. Buenos Aires: Ed. Lumen-Hvmanitas.

(2016). ¿Qué es la economía solidaria? LuisRazeto.net. Argentina: Recuperado de http://www.luisrazeto.net/content/¿quées-la-economí-solidaria

Red de Redes de Economía Alternativa y Solidaria (2011). Carta de Principios de la Economía Solidaria. El portal de economía solidaria. España. Recuperado de http://www.economiasolidaria.org/carta.php

Secretaría de Cultura (2016). Muestra Internacional de Arquitectura de la Bienal de Venecia México. México: Secretaría de Cultura/Instituto Nacional de Bellas Artes.

Sousa Santos, B. de (2010). Refundación del Estado en América Latina: perspectivas desde una epistemología del Sur. México: Siglo XXI/Siglo del Hombre Editores/Universidad de los Andes. 